\title{
Aporte calórico nutricional en la tonificación muscular: Caso de estudio deportistas semiprofesionales de fútbol
}

Nutritional calorie intake in the muscle training: a semiprofessional soccer players case study

Washington Ernesto Castro Acosta. ${ }^{1}$, José Luis Aimacaña Hinojosa. ${ }^{2}$, Christian Mauricio Sánchez Cañizares. ${ }^{3} \&$ Julio Alfonso Mocha Bonilla. ${ }^{4}$

Recibido: 10-02-2019 / Revisado: 15-02-2019 /Aceptado: 04-03-2019/ Publicado: 14-06-2019

\begin{abstract}
.
DOI: https://doi.org/10.33262/cienciadigital.v3i2.5.537

The present study consists of the application of a caloric contribution program based on the method dissociated by immediate principles in competition microcycle and evaluates its incidence in the muscular toning of the football players of the Technical University of Cotopaxi. The nutritional program was established for six months based on the controlled intake of calories from carbohydrates, fats and proteins, in the muscle toning was considered the indicators of body mass index, body weight and percentage of fat, two measurements were made one prior to the adoption of the program and another at the end of it. The study population is made up of 23 football players between the ages of 18 and 24 . Once the program was applied, it was evaluated and average scores were obtained for the case study, muscle toning indicators show that the body weight in the pre-test was 69.9 and in the post test was 68.8 kilograms, likewise the BMI in the pre was 23.10 and for the post was 22.72 , while the $\%$ of
\end{abstract}

\footnotetext{
${ }^{1}$ Universidad Técnica de Ambato, Facultad de Ciencias Humanas y de la Educación, Carrera de Cultura Física. Pedagogía de la Actividad Física y Deporte. Ambato, Ecuador. we.castro@uta.edu.ec

${ }^{2}$ Universidad Técnica de Ambato, Facultad de Ciencias Humanas y de la Educación, Carrera de Cultura FísicaAmbato, Ecuador. jaimacana0507@uta.edu.ec

${ }^{3}$ Universidad Técnica de Ambato, Facultad de Ciencias Humanas y de la Educación, Carrera de Cultura FísicaPedagogía de la Actividad Física y Deporte. Ambato, Ecuador. cm.sanchezc@uta.edu.ec

${ }^{4}$ Universidad Técnica de Ambato, Facultad de Ciencias Humanas y de la Educación, Carrera de Cultura FísicaPedagogía de la Actividad Física y Deporte. Ambato, Ecuador. ja.mocha@uta.edu.ec
} 
GC in the pre was 12.13 and decreased in the post to 11.12; the t-Student test for related samples showed a change in muscle tone according to the $p$-value $<0.05$ for the studied indicators. For this reason, it is determined that the caloric contribution program was satisfactory in the semi-professional athletes of the Technical University Club of Cotopaxi (UTC).

Keywords: Calorie intake, nutritional program, muscle toning, weight, body fat.

\section{Resumen.}

El presente estudio consiste en la aplicación de un programa de aporte calórico basado en el método disociado por principios inmediatos en microciclo de competición y evalúa su incidencia en la tonificación muscular de los futbolistas de la Universidad Técnica de Cotopaxi. El programa nutricional se estableció para seis meses basado en la ingesta controlada de calorías de hidratos de carbono, grasas y proteínas, en la tonificación muscular se consideró los indicadores del índice de masa corporal, peso corporal y porcentaje de grasa; se realizaron dos mediciones una previo a la adopción del programa y otra al final del mismo. La población de estudio está conformada por 23 futbolistas con edades comprendidas entre 18 y 24 años de edad. Una vez aplicado el programa, se evaluó y se obtuvieron puntuaciones promedio para el caso de estudio, los indicadores de tonificación muscular muestran que el peso corporal en el pre-test fue de 69,9 y en el post test fue de 68,8 kilogramos, de igual manera el IMC en el pre fue de 23,10 y para el post fue de 22,72, mientras que el \% de GC en el pre fue de 12,13 y disminuyo en el post a 11.12; la prueba t-Student para muestras relacionadas demostró un cambio en la tonificación muscular según el p-valor $<0,05$ para los indicadores estudiados. Por tal razón, se determina que el programa de aporte calórico fue satisfactorio en los deportistas semiprofesionales del Club Universidad Técnica de Cotopaxi (UTC).

Palabras claves: Aporte calórico, programa nutricional, tonificación muscular, peso, grasa corporal.

\section{Introducción.}


La prevalencia a nivel mundial en obesidad y sobrepeso reflejado en los datos de la OMS, han hecho reflexionar sobre las actuaciones desde todas las áreas; en especial desde la educación física y el deporte para mejorar los hábitos alimenticios (Gil, 2019), en la competitiva sociedad actual, los hombres buscan aumentar su masa muscular con el objetivo de mejorar su imagen social e incrementar su musculatura (Ruiz Pérez, 2019). El ser humano tiene un determinado metabolismo que depende de sus características genéticas, por consiguiente a esas características se debe adaptar una dieta adecuada (Ozonas, 2016).

La nutrición en los seres humanos constituye el punto de conjunción entre los aspectos psicológico, biológico, y social, al mismo tiempo representa un factor relevante en el rendimiento deportivo. La nutrición en los deportistas aporta la cantidad de energía necesaria para el desarrollo de las tareas y proporciona los nutrientes requeridos para la mantención, reparación y construcción de tejidos nuevos en el cuerpo humano (Van Loon, 2009; Pennings, 2011). Los deportistas de competición demandan la ingesta de una dieta equilibrada, basada en variedad, moderación y equilibrio, principios que se reflejan el aporte calórico ideal, "una combinación de hidratos de carbono, proteínas y grasas" (Pérez-Huerta \& Damas-Gutiérrez, 2016, p. 18).

El fútbol como deporte colectivo es practicado a nivel mundial y tiene gran repercusión social a nivel profesional; mientras que a nivel semi-profesional durante la competición de la segunda categoría del fútbol ecuatoriano, demanda que sus futbolistas se preparen tanto en su forma física y técnica para alcanzar un alto rendimiento; adicionalmente interviene de forma determinante el factor denominado aporte calórico nutricional en el rendimiento deportivo del futbolista, es decir, "la proporción de sustancias alimenticias en su dieta diaria" (Martínez-Reñón \& Sánchez-Collado, 2013, p. 320).

El rendimiento deportivo, involucra el desempeño físico del jugador de fútbol, el cual está estrechamente vinculado a la tonificación muscular, que se define como el grado de tensión intramuscular que un músculo presenta en condiciones de reposo. El tono muscular además cumple una tarea condicionante en la capacidad de contracción muscular, en este sentido, se conoce que las posibilidades de desarrollar lesiones decrecen cuando existen altos grados de 
tonificación muscular. La tonificación muscular y la fuerza están influenciados por la masa muscular, el sexo, la edad y el nivel de entrenamiento (García, Martínez, \& Tabuenca, 2005, p. 25). Las funciones principales de los músculos son producir los movimientos, mantener la postura y estabilizar las articulaciones por medio de los músculos estriados, esqueléticos, lisos, viscerales y el miocardio (Waymel \& Choque, 2004, pp. 18-19).

La presencia de grasa corporal es indispensable en los deportistas, cada futbolistas debe tener un nivel óptimo de grasa corporal, la cual le permite alcanzar su máximo rendimiento deportivo. La masa muscular se mide a través del indicador internacional denominado índice de masa corporal (IMC), en cuanto al porcentaje de grasa corporal (\% GC), se calcula a partir de la densidad corporal, utilizando la fórmula establecida por Siri, (1961), también la de Brozek, Grande, Anderson y Keys (1963).

Durante el período de formación de los jóvenes futbolistas tienen lugar una serie de cambios antropométricos que determinarán su forma física y potencial de rendimiento deportivo, por tanto, la nutrición durante esta etapa de desarrollo puede ser decisiva para el futuro deportista (González-Neira, 2015). Los avances de la nutrición revelan la importancia que tiene la alimentación adecuada sobre el bienestar físico, emocional y el rendimiento en el campo del fútbol (Suárez, 2016). La composición corporal en el fútbol consiste principalmente en el seguimiento de la grasa corporal y masa muscular. Mantener la grasa corporal dentro de un rango óptimo puede conservar la relación potencia / masa, lo que permite más movimiento eficiente durante el entrenamiento y los partidos (Collins, 2014).

En la publicación de la FIFA (2005) titulada "F-MARC Nutrición para el fútbol se presenta una guía práctica para comer y beber a fin de mejorar el rendimiento y la salud", se definen algunas estrategias para ayudar a la planificación de las comidas durante la semana para los futbolistas; la cual sugiere planificar el menú semanal alrededor de los partidos y sesiones de entrenamiento, llevando un registro del día a día en el que el aporte calórico tiene que ajustarse al horario de entrenamiento, la competencia y el desarrollo físico del deportista, tomando en cuenta las demandas de energía con respecto al ritmo de trabajo y la intensidad del ejercicio, según el libro de la FIFA basada en la conferencia Internacional de consenso 
en la sede de la (FIFA, 2005) en Gutiérrez Muñoz, (2018). En este sentido, es fundamental “determinar las cantidades de hidratos de carbono, proteínas, grasas, vitaminas, minerales y antioxidantes que debe ingerir el deportista" (Pérez-Huerta \& Damas-Gutiérrez, 2016, pp. 21-28). Por otra parte, según González, Cobos y Molina (2010), en su estudio denominado "Estrategias Nutricionales para la Competición en el Fútbol”, recomiendan establecer una estructura de dieta disociada en microciclo de competición, la cual establece una distribución calórica de la dieta pre-partido en función de la hora de los partidos, es decir, la dieta para los partidos que se efectúan en la mañana, tarde o noche, sin dejar de lado la forma de hidratación y el aporte nutricional intrapartido. Se denomina método disociado de aporte calórico por principios inmediatos porque se formula para ocho días en cada ciclo, de los cuales los seis primeros son previos al desarrollo del partido, el séptimo es el día del encuentro y el octavo es el siguiente día del evento deportivo (pp. 119-120).

La nutrición en la disciplina del fútbol siempre ha sido una de las últimas prioridades cuando los entrenadores y médicos del equipo preparan a los jugadores para la competición, en términos literales, el nutricionista deportivo es quien puede, de hecho, causar el mayor impacto en la "formación” de un equipo exitoso (Rollo, 2018), por consiguiente el propósito es analizar el efecto de un programa de aporte calórico nutricional en la tonificación muscular de los futbolistas del Club Universidad Técnica de Cotopaxi (UTC).

\section{Metodologia.}

El estudio es de tipo cuasi-experimental, se tomó un grupo de individuos en función de una nómina establecida, deportistas seleccionados del club Universidad Técnica de Cotopaxi, considerado como grupo de intervención, el cual fue sometido al programa de aporte calórico nutricional, se efectuaron dos mediciones, una medición previa y una posterior, por lo tanto, se trata de un estudio de tipo longitudinal "antes-después", por el hecho de que se calcularon las variables en dos ocasiones mediante la utilización de un test antropométrico. Se utilizó un enfoque cuantitativo, ya que se midió los indicadores de tonificación muscular: peso, índice de masa corporal y porcentaje de grasa corporal. La investigación tiene como modalidad un trabajo de campo, dado que los investigadores acudieron directamente al complejo de entrenamiento de Club UTC, para supervisar la adopción del programa de aporte 
calórico nutricional ejecutado en seis meses a cada uno de los integrantes, realizando las mediciones correspondientes para evaluar la tonificación muscular.

\section{Sujetos}

Los participantes del estudio fueron 23 futbolistas del Club Universidad Técnica de Cotopaxi, que participan en el torneo provincial de Segunda Categoría, los participantes son de sexo masculino, cuyo promedio y desviación estándar de edad, talla y peso son: $22.09 \pm 2.38$ años de edad; $1.74 \pm 0.05 \mathrm{~m}$ de altura; y $69.87 \pm 4.86 \mathrm{~kg}$ de peso, respectivamente. Los futbolistas que participaron en el estudio recibieron una notificación previa a través de una carta de participación, en la que se informó acerca de los objetivos del estudio, las condiciones del aporte nutricional, la forma en la que serán supervisados y evaluados. De esta manera, todos los participantes expresaron su consentimiento voluntario de participar del estudio.

\section{Instrumentos}

Se evaluó la tonificación muscular de los futbolistas, tomando en cuenta los siguientes indicadores:

- Índice de masa corporal (IMC), calculado mediante la siguiente fórmula:

$$
\mathrm{IMC}=\frac{\text { Masa corporal }(\mathrm{kg})}{\text { talla }(\mathrm{m}) \times \text { talla }(\mathrm{m})}
$$

- La densidad corporal (DC) se determina a través de la fórmula establecida por Durnin/Womersley (1974) para 4 pliegues:

$$
\mathrm{DC}=\mathrm{C}-[M \times \log (\text { suma de pliegues) }]
$$

suma de pliegues $(\mathrm{mm})=$ Bicep + Tricep + Subescapular + Suplailiaco

M y C son constantes que dependen de la edad y sexo de la persona. Para hombres de 18 a 29 años de edad son $\mathrm{C}=1.1631 ; \mathrm{M}=0.0632$.

- El porcentaje de grasa corporal (\% GC) se calcula con la ecuación propuesta por Siri (1961): 


$$
\% \mathrm{GC}=\left[\left(\frac{4,95}{\mathrm{DC}}\right)-4,5\right] \times 100 \%
$$

Los instrumentos de medición utilizados fueron una balanza, un tallímetro, y un plicómetro o lipocalibre.

Los datos establecidos durante el trabajo de campo en dos momentos (pre y post), permitieron obtener la información necesaria para aplicar una prueba t-Student para muestras relacionadas, comprobar la hipótesis y establecer las conclusiones respectivas, concordando de esta manera con Arancibia, (2016) quien menciona que se debe aplicar mediciones antropométricas para obtener Índice de Masa Corporal (IMC) y el porcentaje de grasa corporal $(\% \mathrm{GC})$.

\section{Protocolos}

\section{Aplicación del programa de nutrición}

El programa de aporte calorico nutricional establecido tiene como referente al "Método disociado de aporte calórico por principios inmediatos en microciclo de competición", desarrollado por González, Cobos y Molina (2010), para el presente estudio tuvo una duración de seis meses, tomando en cuenta lo mencionado por (García, 2018) se realiza una medición inicial (MI) y después de 6 meses una medición final (MF), y se aplica una prueba t-student para valorar la masa magra, de igual manera se toma en cuenta lo expuesto por Martínez-Reñón y Sánchez-Collado (2013) para establecer dos microciclo de tres meses de duración, aplicando un calendario semanal organizado, tomando en cuenta que en el último día se desarrolla el partido oficial de fútbol.

- El balance energético en función del tipo de día del programa de aporte nutricional se establece considerando los días de descanso, los de entrenamiento y el día de competición. Tomando en consideración dichos parámetros se determinan los valores energéticos en kilocalorías (kcal) que deben ser ingeridos por los deportistas. Para mayor detalle se presenta la Tabla 1.

Tabla 1. Balance energético en función del tipo de (Día).

\begin{tabular}{cccccccc} 
Valores de & \multicolumn{8}{c}{ Día } \\
\cline { 2 - 8 } ingesta & Descanso & $\mathbf{2}$ & $\mathbf{3}$ & $\mathbf{4}$ & $\mathbf{5}$ & $\mathbf{6}$ & Competición \\
& $\mathbf{1}$ & $\mathbf{2}$ & 3570 & 3570 & 3570 & 3570 & $\mathbf{7}$ \\
Energía & 3080 & 3570 & 3500 \\
(kcal) & $100 \%$ & $100 \%$ & $100 \%$ & $100 \%$ & $100 \%$ & $100 \%$ & $100 \%$
\end{tabular}


Valores de ingesta

Hidratos de carbono

(kcal)

$$
\%
$$

Grasas

(kcal)

$\%$

Proteínas

(kcal)

$\%$

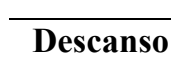

1

1540

$50 \%$

924

$30 \%$

616

$20 \%$

Día

\section{Entrenamiento}

2

2678

$75 \%$

$15 \%$

357

$10 \%$
3

2678

$75 \%$

2678

$75 \%$

535

$15 \%$

4

535

$15 \%$

357

$10 \%$

$10 \%$

(1)

357

5

2678

$75 \%$

2678

2100

$60 \%$

Competición

7

$\begin{array}{ll}6 & 7\end{array}$

$75 \%$

875

$25 \%$

$15 \% \quad 15 \%$

357

357

525

$10 \%$

$10 \%$

$15 \%$

Fuente: Elaboración propia.

Para la valoración dietética se elaboraron menús cíclicos planificados por cada día, con el objeto de garantizar el aporte calórico de entre 3080 y $3570 \mathrm{kcal}$, cumpliendo de esta manera con el requerimiento nutricional aproximado para cada futbolista. Durante el desarrollo del encuentro futbolístico un aspecto fundamental es la hidratación y el aporte nutricional intrapartido, regularmente la mayoría de los futbolistas no ingieren una cantidad de líquidos suficiente, antes, durante y después en función de las condiciones climáticas y de la intensidad del partido, las pérdidas por sudoración en los futbolistas pueden ir de 1 a 4 litros. El uso de bebidas deportivas comerciales con un contenido aproximado de 4 a $8 \%$ de carbohidratos (4 a $8 \mathrm{~g} / 100 \mathrm{ml}$ ) permiten cubrir al mismo tiempo las necesidades de carbohidratos y fluidos en casi todos los casos.

\section{Procedimiento para la evaluación de la tonificación muscular}

Los indicadores de medición de la tonificación muscular en los futbolistas se evaluaron de la siguiente manera:

- El índice de masa corporal (IMC) se determina empleando la fórmula (1), para lo cual se empleó una balanza y un tallimetro; mediante un indicador sencillo de medición, su cálculo es el resultado de la división entre el peso en kilogramos por el cuadrado de su tamaño en metros $(\mathrm{kg} / \mathrm{m} 2)$, cuyo resultado final se aplica para obtener el (IMC) de una persona (Mocha-Bonilla, 2018) tanto en el pre-test como en el post-test. 
- La densidad corporal (DC) se determinó a través de la fórmula (2) de Durnin/Womersley (1974) para 4 pliegues. Se midieron los pliegues: biceps, triceps, subescapular y suprailíaco; lo que permitió determinar el valor de la suma de pligues utilizando la fórmula (3). Se empleó un plicómetro para la medición de los pliegues indicados.

- El porcentaje de grasa corporal (\% GC) se calculó por medio de la fórmula (4) propuesta por Siri (1961), con base en los datos de densidad corporal previamente obtenidos. "Para corroborar los datos obtenidos se utilizó un plicómetro/lipocalibre según menciona” (PérezHuerta \& Damas-Gutiérrez, 2016, pp. 40-41).

\section{Resultados.}

Los valores calculados de los indicadores de la tonificación muscular de los futbolistas del club UTC, tanto en el pre-test como en el post-test se muestran en la Tabla 2.

Tabla 2. Datos de los indicadores de tonificación muscular del pre-test y post-test.

\begin{tabular}{|c|c|c|c|c|c|c|c|c|c|c|c|}
\hline \multirow{2}{*}{$\begin{array}{c}\mathbf{N}^{\mathbf{o}} \\
\text { FUTBOLIS } \\
\text { TA-UTC }\end{array}$} & \multicolumn{2}{|c|}{$\begin{array}{l}\text { PARÁMETROS } \\
\text { BÁSICOS }\end{array}$} & \multicolumn{3}{|c|}{$\begin{array}{c}\text { PESO } \\
\text { CORPORAL (kg) }\end{array}$} & \multicolumn{3}{|c|}{$\begin{array}{l}\text { ÍNDICE DE MASA } \\
\text { CORPORAL IMC }\end{array}$} & \multicolumn{3}{|c|}{$\begin{array}{c}\text { PORCENTAJE DE } \\
\text { GRASA CORPORAL (\%) }\end{array}$} \\
\hline & $\begin{array}{l}\text { Ed } \\
\text { ad }\end{array}$ & $\begin{array}{c}\text { Estatura } \\
(\mathbf{m})\end{array}$ & $\begin{array}{l}\text { Pre- } \\
\text { Test }\end{array}$ & $\begin{array}{l}\text { Post- } \\
\text { Test }\end{array}$ & $\begin{array}{l}\text { Diferen } \\
\text { cias }\end{array}$ & $\begin{array}{l}\text { Pre- } \\
\text { Test }\end{array}$ & $\begin{array}{l}\text { Post- } \\
\text { Test }\end{array}$ & $\begin{array}{l}\text { Diferen } \\
\text { cias }\end{array}$ & $\begin{array}{l}\text { Pre- } \\
\text { Test }\end{array}$ & $\begin{array}{l}\text { Post- } \\
\text { Test }\end{array}$ & $\begin{array}{l}\text { Diferen } \\
\text { cias }\end{array}$ \\
\hline 1 & 20 & 1,74 & 75 & 73 & 2 & 24,77 & 24,11 & 0,66 & 10,57 & 8,53 & 2,04 \\
\hline 2 & 24 & 1,69 & 65 & 63 & 2 & 22,76 & 22,06 & 0,70 & 10,09 & 9,59 & 0,50 \\
\hline 3 & 22 & 1,78 & 76 & 74 & 2 & 23,99 & 23,36 & 0,63 & 15,23 & 14,56 & 0,67 \\
\hline 4 & 21 & 1,71 & 64 & 63 & 1 & 21,89 & 21,55 & 0,34 & 13,49 & 12,32 & 1,17 \\
\hline 5 & 19 & 1,75 & 72 & 71 & 1 & 23,51 & 23,18 & 0,33 & 11,48 & 9,59 & 1,89 \\
\hline 6 & 22 & 1,65 & 64 & 63 & 1 & 23,51 & 23,14 & 0,37 & 8,53 & 7,37 & 1,16 \\
\hline 7 & 23 & 1,73 & 65 & 63 & 2 & 21,72 & 21,05 & 0,67 & 9,07 & 7,37 & 1,70 \\
\hline 8 & 23 & 1,88 & 81 & 79 & 2 & 22,92 & 22,35 & 0,57 & 16,17 & 14,9 & 1,27 \\
\hline 9 & 24 & 1,72 & 73 & 75 & -2 & 26,18 & 26,89 & $-0,71$ & 11,91 & 11,48 & 0,43 \\
\hline 10 & 20 & 1,71 & 69 & 69 & 0 & 23,32 & 23,32 & 0,00 & 13,12 & 11,91 & 1,21 \\
\hline 11 & 22 & 1,75 & 71 & 71 & 0 & 23,18 & 23,18 & 0,00 & 13,86 & 13,12 & 0,74 \\
\hline 12 & 18 & 1,77 & 73 & 71 & 2 & 23,3 & 22,34 & 0,96 & 14,56 & 14,22 & 0,34 \\
\hline 13 & 21 & 1,66 & 65 & 66 & -1 & 23,59 & 23,95 & $-0,36$ & 9,59 & 9,59 & 0,00 \\
\hline 14 & 23 & 1,70 & 69 & 67 & 2 & 23,88 & 23,18 & 0,70 & 11,48 & 11,03 & 0,45 \\
\hline 15 & 19 & 1,80 & 74 & 73 & 1 & 22,84 & 22,53 & 0,31 & 12,32 & 11,03 & 1,29 \\
\hline 16 & 20 & 1,75 & 72 & 70 & 2 & 23,51 & 22,2 & 1,31 & 10,09 & 9,59 & 0,50 \\
\hline 17 & 24 & 1,81 & 76 & 73 & 3 & 23,2 & 22,28 & 0,92 & 14,9 & 14,22 & 0,68 \\
\hline 18 & 22 & 1,83 & 67 & 68 & -1 & 20,01 & 20,31 & $-0,30$ & 13,86 & 13,12 & 0,74 \\
\hline 19 & 24 & 1,73 & 73 & 71 & 2 & 24,39 & 23,72 & 0,67 & 13,86 & 13,12 & 0,74 \\
\hline 20 & 20 & 1,69 & 65 & 63 & 2 & 22,76 & 22,06 & 0,70 & 12,32 & 11,03 & 1,29 \\
\hline 21 & 24 & 1,71 & 63 & 63 & 0 & 21,2 & 21,55 & $-0,35$ & 8,53 & 6,74 & 1,79 \\
\hline
\end{tabular}




\begin{tabular}{|c|c|c|c|c|c|c|c|c|c|c|c|}
\hline \multirow{2}{*}{$\begin{array}{c}\mathbf{N}^{\mathbf{0}} \\
\text { FUTBOLIS } \\
\text { TA-UTC }\end{array}$} & \multicolumn{2}{|c|}{$\begin{array}{l}\text { PARÁMETROS } \\
\text { BÁSICOS }\end{array}$} & \multicolumn{3}{|c|}{$\begin{array}{c}\text { PESO } \\
\text { CORPORAL (kg) }\end{array}$} & \multicolumn{3}{|c|}{$\begin{array}{l}\text { ÍNDICE DE MASA } \\
\text { CORPORAL IMC }\end{array}$} & \multicolumn{3}{|c|}{$\begin{array}{l}\text { PORCENTAJE DE } \\
\text { GRASA CORPORAL (\%) }\end{array}$} \\
\hline & $\begin{array}{l}\text { Ed } \\
\text { ad }\end{array}$ & $\begin{array}{c}\text { Estatura } \\
(\mathbf{m})\end{array}$ & $\begin{array}{l}\text { Pre- } \\
\text { Test }\end{array}$ & $\begin{array}{l}\text { Post- } \\
\text { Test }\end{array}$ & $\begin{array}{c}\text { Diferen } \\
\text { cias }\end{array}$ & $\begin{array}{l}\text { Pre- } \\
\text { Test }\end{array}$ & $\begin{array}{l}\text { Post- } \\
\text { Test }\end{array}$ & $\begin{array}{l}\text { Diferen } \\
\text { cias }\end{array}$ & $\begin{array}{l}\text { Pre- } \\
\text { Test }\end{array}$ & $\begin{array}{l}\text { Post- } \\
\text { Test }\end{array}$ & $\begin{array}{c}\text { Diferen } \\
\text { cias }\end{array}$ \\
\hline 22 & 24 & 1,76 & 65 & 65 & 0 & 20,98 & 20,98 & 0,00 & 13,49 & 12,32 & 1,17 \\
\hline \multirow[t]{2}{*}{23} & 23 & 1,72 & 71 & 69 & 2 & 24 & 23,32 & 0,68 & 10,57 & 9,07 & 1,50 \\
\hline & $\begin{array}{c}21 \\
8\end{array}$ & 1,74 & 69,9 & 68,8 & 1,1 & 23,10 & 22,72 & 0,38 & 12,13 & 11,12 & 1,01 \\
\hline
\end{tabular}

Fuente: Elaboración propia.

\section{Estadísticos descriptivos pre-test vs post-test}

A partir de los datos mostrados en la Tabla 3 se obtuvieron los siguientes estadísticos descriptivos, para cada uno de los indicadores establecidos:

Tabla 3. Estadísticos descriptivos para muestras emparejadas.

\begin{tabular}{cccccc}
\multicolumn{2}{c}{ Indicador de Tonificación Muscular } & Media & N & Desviación estándar & $\begin{array}{c}\text { Error Estándar } \\
\text { de la media }\end{array}$ \\
\hline Peso Corporal (kg) & Pre-Test & 69.87 & 23 & 4.966 & 1.036 \\
& Post-Test & 68.70 & 23 & 4.607 & 0.961 \\
Índice de Masa Corporal & Pre-Test & 23.10 & 23 & 1.3449 & 0.280 \\
IMC & Post-Test & 22.72 & 23 & 1.3203 & 0.275 \\
Porcentaje de Grasa & Pre-Test & 12.13 & 23 & 2.4176 & 0.504 \\
Corporal (\%) & Post-Test & 11.12 & 23 & 2.2265 & 0.464
\end{tabular}

Fuente: Elaboración propia.

Como se observa en la Tabla 5, en los casos del IMC y el \% GC, las medias obtenidas en el pre-test son de mayor magnitud en relación a las del post-test; lo cual son indicadores positivos, dado que reflejan que el programa de aporte calórico nutricional permitió disminuir las puntuaciones en la composición corporal de los sujetos de estudio. Sin embargo, para conocer si el mejoramiento es significativo se aplicó la prueba estadística t-Student para muestras relacionadas por tratarse de un estudio comparativo (pre $=$ antes; post $=$ después de realizar un programa de Intervención=Plan de aporte calórico nutricional en la mejora de la tonificación muscular. A continuación se presentan los resultados.

Posterior a la adopción del programa de aporte calórico nutricional se establece que, el IMC, presenta valores de 69,9 en el pre-test, mientras que para el post-test se obtuvo 68,8. Al comparar directamente cada uno de los datos, como se observa en el figura 1, se aprecia que el IMC en el post-test desciende ligeramente en la mayoría de los casos, mediante la prueba estadística se demostró que esta diferencia es significativa. 


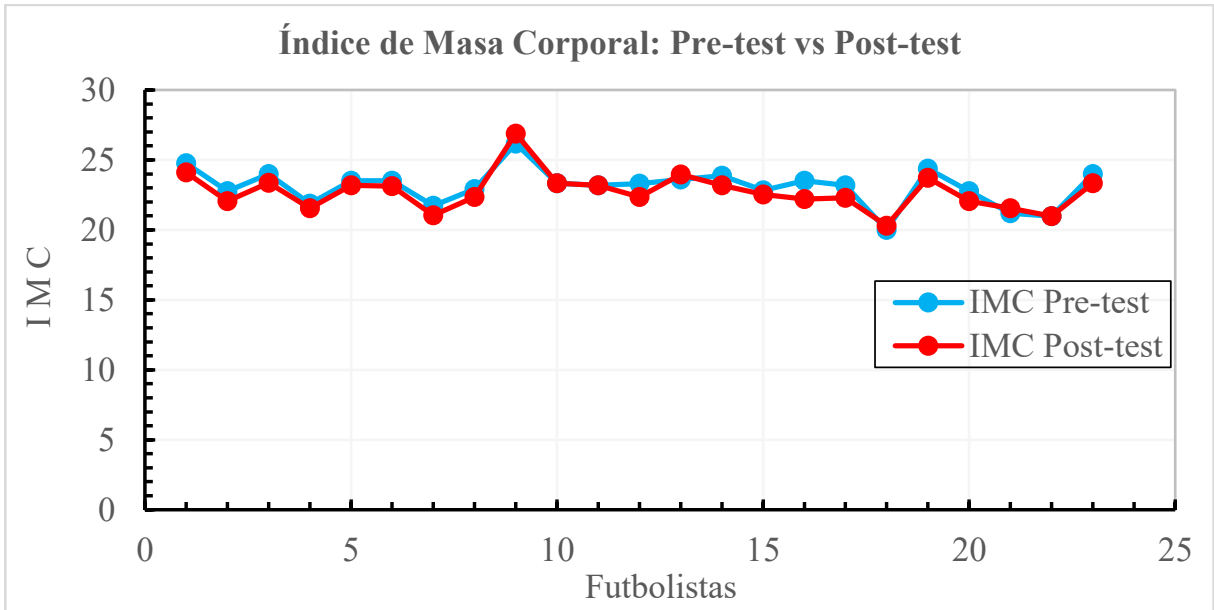

Figura 1. Índice de masa corporal pre-test y post-test.

Fuente: Elaboración propia.

Al referirse al peso corporal, durante el pre-test el valor es de 23,10 kg; mientras que en el post-test se obtuvo un valor de $22,72 \mathrm{~kg}$. Al comparar todos los datos, conforme el figura 2 , se observa diferencia entre el pre y el post test manteniendo valores equivalentes a un IMC Normal.

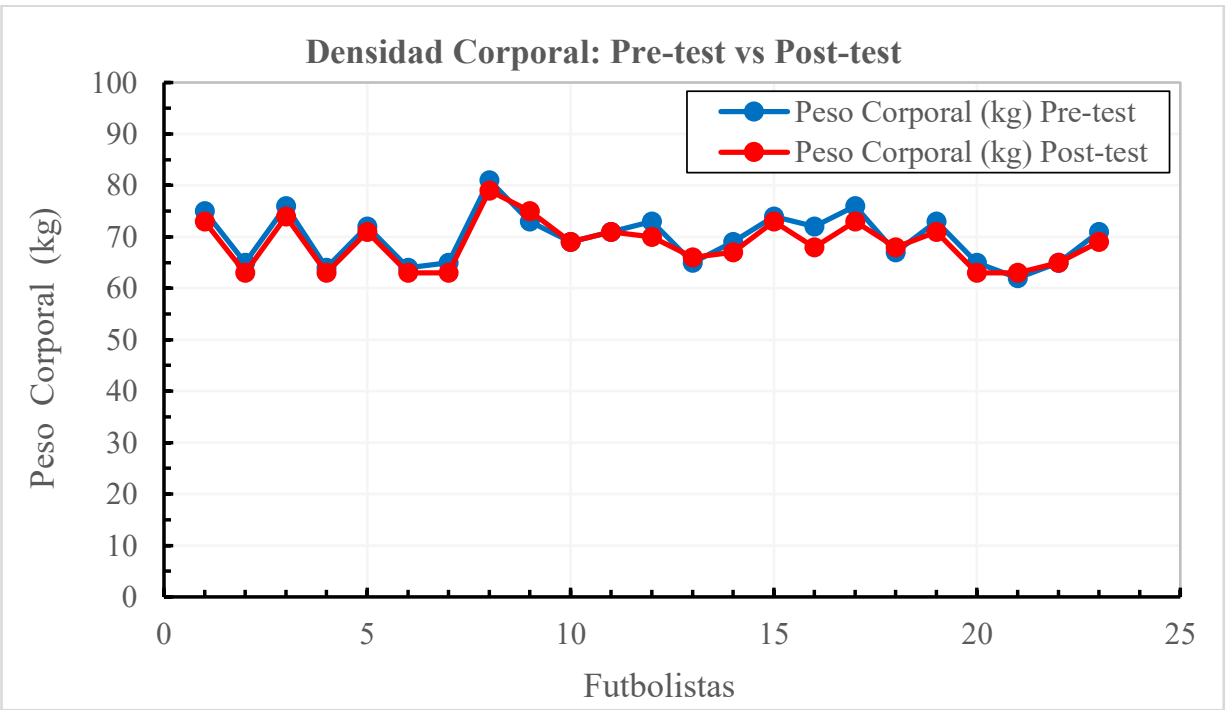

Figura 2. Densidad corporal en el pre-test y post-test. Fuente: Elaboración propia.

El porcentaje de grasa corporal presenta valores dispersos entre los participantes del estudio, tal es así que en el pre test se presenta un 12,13\%; mientras que el rango bajo a un 11,12\% 
en el post-test. Mediante el figura 3 se puede apreciar una disminución del porcentaje de grasa.

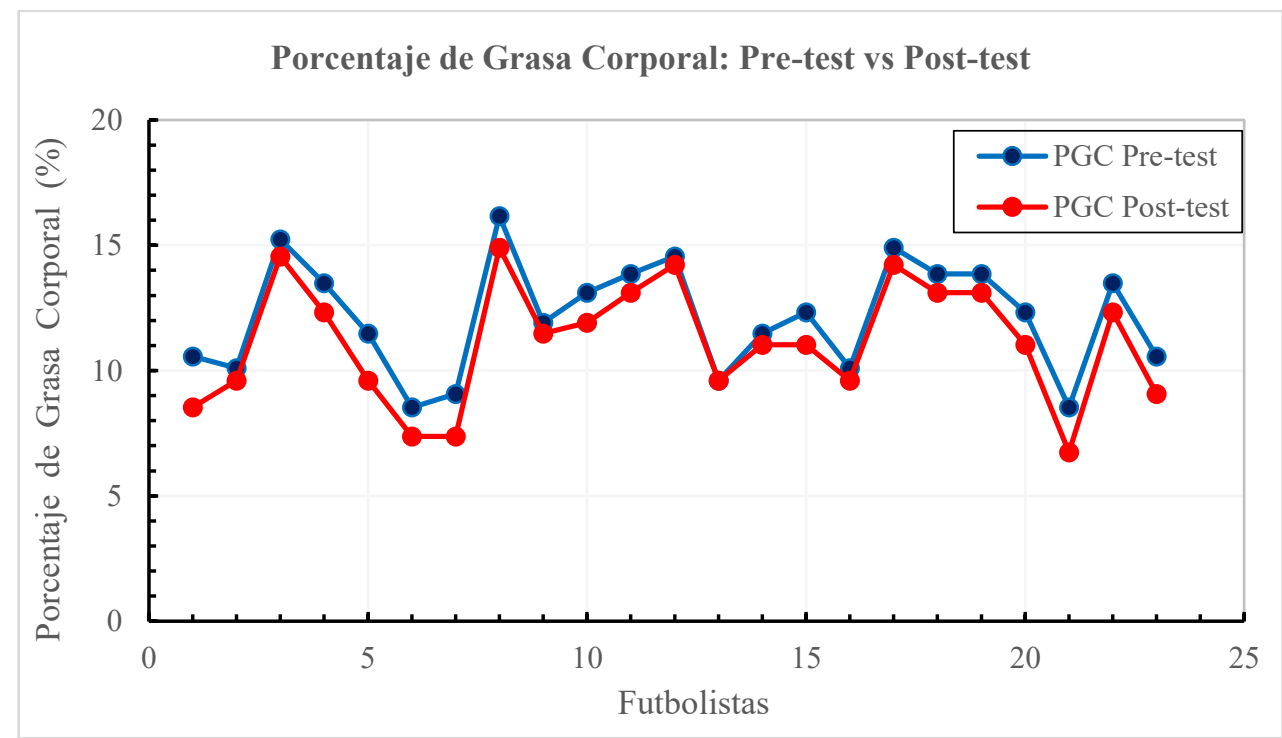

Figura 3. Porcentaje de grasa corporal en el pre-test y post-test.

Fuente: Elaboración propia.

\section{Análisis Estadístico}

Los datos obtenidos de los tres indicadores de la tonificacion muscular de los futbolistas del Club UTC son de tipo numérico. Es por ello que se determina los estadísticos descriptivos: media aritmética, desviación estándar y la media de error estándar. También se realizó el estudio comparativo de los datos obtenidos en el pre-test y post-test, cuya hipótesis de trabajo fue verificar si el programa de aporte calórico nutricional disminuye las puntuaciones en los indicadores de tonificación muscular de los futbolistas del Club Universidad Técnica de Cotopaxi (UTC) mediante una prueba estadística t-Student para muestras realacionadas.

\section{Verificación de hipótesis}

El modelo matemático de la prueba t-Student es comparativo entre las medias de las diferencias de los indicadores de tonificación muscular, antes y después del programa de aporte calórico nutricional, el nivel de significancia admisible es de 0.05 (5\%), con un nivel de confianza del $95 \%$. Una vez calculada la t-Student se muestran los resultados obtenidos a continuación:

Tabla 4. Prueba t-Student para muestras relacionadas y significancia bilateral. 


\begin{tabular}{|c|c|c|c|c|c|c|c|c|c|}
\hline & & & Difere & cias empa & ejadas & & & & \\
\hline & & & & $\begin{array}{l}\text { Media de } \\
\text { error }\end{array}$ & $\begin{array}{r}95 \% \text { de } \\
\text { de confi } \\
\text { dife }\end{array}$ & $\begin{array}{l}\text { intervalo } \\
\text { inza de la } \\
\text { encia }\end{array}$ & & & Sig. \\
\hline & & Media & estándar & estándar & Inferior & Superior & $\mathbf{t}$ & gl & (bilateral) \\
\hline & $\begin{array}{l}\text { Peso }(\mathrm{kg}) \text { Pre-Test } \\
\text { Peso }(\mathrm{kg}) \text { Post-Test }\end{array}$ & 1.1740 & 1.4970 & 0.312 & 0.527 & 1.821 & 3.761 & 22 & 0.001079 \\
\hline & $\begin{array}{l}\text { IMC Pre-Test } \\
\text { IMC Post-Test }\end{array}$ & 0.3826 & 0.4958 & 0.103 & 0.168 & 0.597 & 3.701 & 22 & 0.001248 \\
\hline $\begin{array}{c}\text { Par } \\
1\end{array}$ & $\begin{array}{c}\text { Porcentaje GC }(\%) \\
\text { Pre-Test } \\
\text { Porcentaje GC }(\%) \\
\text { Post-Test }\end{array}$ & 1.1170 & 0.5694 & 0.114 & 0.175 & 1.048 & 3.872 & 22 & 0.020008 \\
\hline
\end{tabular}

Fuente: Elaboración propia.

Decisión: El análisis estadístico realizado mediante la prueba $t$-Student calculados para 22 grados de libertad, en los tres casos se localizan en la región de rechazo, lo que se corrobora en función del margen de significancia ( $p$-valor), que es menor que el margen admisible de 0,05 (5\%). Por tal razón, se acepta la hipótesis de trabajo, en consecuencia: El programa de aporte calórico nutricional disminuyó las puntuaciones en los indicadores de tonificación muscular de los futbolistas del Club Universidad Técnica de Cotopaxi (UTC).

\section{Conclusiones.}

La presente investigación permitió conocer el efecto que produce un programa de aporte calórico en los indicadores de tonificación muscular mediante la implementación de un programa nutricional basado en el método disociado de aporte calórico por principios inmediatos en microciclo de competición, considerando la ingesta controlada de calorías de hidratos de carbono, grasas y proteínas.

La tonificación muscular se evaluó a través de la medición en dos ocasiones, por medio de tres indicadores, entre los que constan el índice de masa corporal, el peso corporal y el porcentaje de grasa. La primera medición se efectuó previo al inicio del programa de nutrición y la segunda fue posterior a la finalización (seis meses). Los resultados demostraron una disminución significativa de los tres indicadores, por lo que el programa de nutrición fue 
satisfactorio, considerando la condición de los participantes, es decir, es significativa para deportistas semiprofesionales en edad competitiva para la disciplina de fútbol.

Otros de los factores considerados en el programa de nutrición fue la valoración dietética aplicada en cuatro momentos: desayuno, refrigerio de media mañana, almuerzo, refrigerio de media tarde y cena; de igual manera aunque se brindó una hidratación ante, durante y después de los entrenamientos y la competencia no se valoró esta variable, la cual es motivo de estudios posteriores.

Mediante la aplicación del estadístico t de Student para muestras relacionadas, se determinó que existen pequeñas diferencias significativas en la disminución de los indicadores evaluados referente a la tonificación muscular, al bajar el peso corporal ayuda a bajar la grasa corporal y mejora la tonificación muscular, sin embargo, hay que tener presente siempre una buena preparación tanto física como psicológica.

\section{Referencias bibliográficas.}

Arencibia-Moreno, R., Hernández-Gallardo, D., \& Paucar-Iza, V. (2016). Estado nutricional y hábitos alimentarios de futbolistas amateur, categoría Sénior, Serie A de la Liga Cantonal Rumiñahui, Ecuador. Nutrición Clínica y Dietética Hospitalaria, 36(3), 114121. https://doi.org/10.12873/363arencibia

Collins, J. \&. (2014). Practical considerations in elite football. Sports Science Exchange, $27(133), 1-7$.

FIFA. (2005). F-MARC Nutrición para el fútbol. Una guía práctica para comer y beber a fin de mejorar el rendimiento y la salud. Recuperado de https://img.fifa.com/image/upload/so6ov0b2xuwh8nupxuea.pdf

García, N., Martínez, A., \& Tabuenca, A. (2005). La tonificación muscular (Teoría y práctica) (3. ${ }^{\mathrm{a}}$ ed.). Badalona, España: Paidotribo.

García, R. L. (2018). Seguimiento de la masa grasa y masa magra por segmentos corporales (brazos y piernas) en futbolistas juveniles monitoring fat mass and lean mass by body segments (arms and legs) in youth soccer players. REVISTA INTERNACIONAL DE DEPORTES COLECTIVOS, 14-16. 
Gil, J. F. (2019). El paradigma de la alimentación saludable en educación primaria.¿ nos estamos equivocando? EmásF: revista digital de educación física(56), 13-29.

González, J. A., Cobos, I., \& Molina, E. (2010). Estrategias Nutricionales para la Competición en el Fútbol. Revista Chilena de Nutrición, 37(1), 118-123. https://doi.org/10.4067/S0717-75182010000100012

González-Neira, M., San Mauro-Martín, I., García-Angulo, B., Fajardo, D., \& GaricanoVilar, E. (2014). Valoración nutricional, evaluación de la composición corporal y su relación con el rendimiento deportivo en un equipo de fútbol femenino. Revista Española de Nutrición Humana y Dietética, 19 (1), 36-48. https://doi.org/10.14306/renhyd.19.1.109

Gutiérrez Muñoz, M. A. (2018). Alimentación en base a nutrición deportiva, mejora de rendimiento para jugadoras de fútbol profesional en el Ecuador de edades comprendidas entre 17 a 30 años del equipo espuce de la ciudad de Quito. Quito: (Bachelor's thesis, QUITO/UIDE/2018).

Martínez-Reñón, C., \& Sánchez-Collado, P. (2013). Estudio nutricional de un equipo de fútbol de tercera división. Nutrición Hospitalaria, 28(2), 319-324. https://doi.org/10.3305/nh.2013.28.2.6304

Mocha-Bonilla, J. A. (2018). Obesity and sedentarism levels analysis: a case study universidad técnica de ambato-ingahurco campus. Ponte International Journal of Sciences and Research, 74(5), 70-83. doi:DOI: 10.21506/j.ponte.2018.5.7

Ozonas, B. R. (2016). Restricción calórica y longevidad. In Anales de la Real Academia Nacional de Farmacia (Vol. 82)., 82, 76-86.

Pérez-Huerta, R., \& Damas-Gutiérrez, N. (2016). Programa Nutricional para Jugadores de Fútbol del Club Jaguares de Chiapas (Tesis de Pregrado). Universidad de Ciencias y Artes de Chiapas, Tuxtla Gutiérrez, Chiapas.

Rollo, I. \&. (17 de febrero de 2018). Nutrición para la recuperación en el fútbol: la formación de un equipo. Obtenido de Nutrición: https://barcainnovationhub.com/es/nutricion-para-recuperacion-futbol/

Ruiz Pérez, S. (2019). Estudio intercultural de la distribución calórico-dietética y su impacto en la autopercepción corporal en varones no competidores de musculación. España: (Doctoral dissertation, Universitat Ramon Llull). 
Suárez, M. L. (2016). Alimentación, suplementación y composición corporal de jugadoras de fútbol femenino de primera división de la ciudad de Córdoba. Cordova: (Bachelor's thesis).

Waymel, T., \& Choque, J. (2004). 250 Ejercicios de estiramiento y tonificación muscular (3. a ed.). Barcelona, España: Paidotribo.

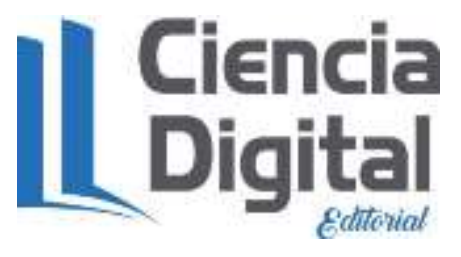

\section{PARA CITAR EL ARTÍCULO INDEXADO.}

Castro Acosta, W., Aimacaña Hinojosa, J., Sánchez Cañizares, C., \& Mocha Bonilla, J. (2019). Aporte calórico nutricional en la tonificación muscular: Caso de estudio deportistas semiprofesionales de fútbol. Ciencia Digital, 3(2.5), 134-149. https://doi.org/10.33262/cienciadigital.v3i2.5.537

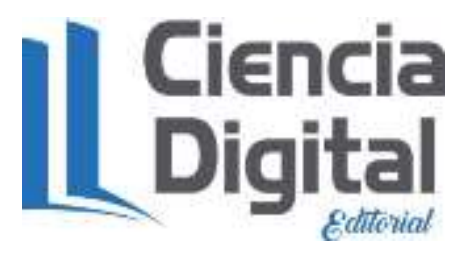

El artículo que se publica es de exclusiva responsabilidad de los autores y no necesariamente reflejan el pensamiento de la Revista Ciencia Digital.

El artículo queda en propiedad de la revista y, por tanto, su publicación parcial y/o total en otro medio tiene que ser autorizado por el director de la Revista Ciencia Digital.
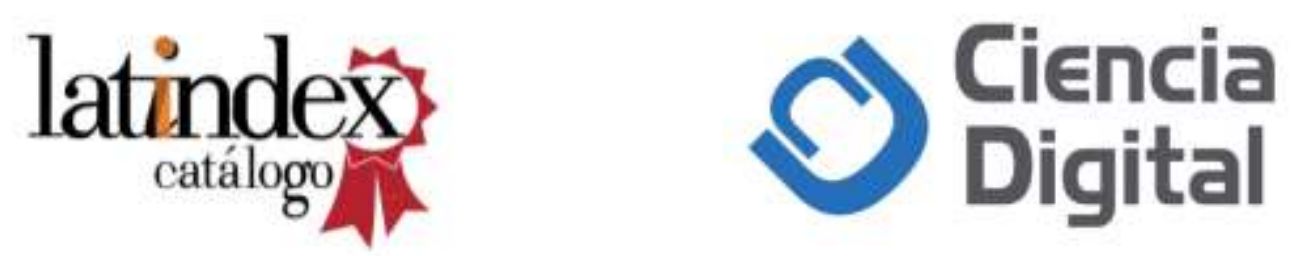